University of Wollongong

Research Online

Faculty of Engineering and Information

Faculty of Engineering and Information

Sciences - Papers: Part A

Sciences

$1-1-2012$

Study on surface roughness and friction during hot rolling of stainless steel 301

Dongbin Wei

University of Wollongong, dwei@uow.edu.au

Zhengyi Jiang

University of Wollongong, jiang@uow.edu.au

Junxia Huang

Baosteel

Aiwen Zhang

az483@uow.edu.au

Xu Shi

Baosteel

See next page for additional authors

Follow this and additional works at: https://ro.uow.edu.au/eispapers

Part of the Engineering Commons, and the Science and Technology Studies Commons

Research Online is the open access institutional repository for the University of Wollongong. For further information contact the UOW Library: research-pubs@uow.edu.au 


\title{
Study on surface roughness and friction during hot rolling of stainless steel 301
}

\author{
Abstract \\ A well-defined boundary condition is important for generating an accurate model for simulating metal \\ forming process. It is important to characterize the features of the oxide scale in hot rolling of stainless \\ steel strip. Short time oxidation tests in humid air with water vapor content of 7.0 vol. \% were carried out \\ using Gleeble 3500 thermo-mechanical simulator. The deformation, surface morphology of oxide scale, \\ and the friction in hot rolling were studied by conducting hot rolling tests. The results show that the \\ surface roughness decreases with an increase of reduction. The effect of oxide scale on friction and \\ surface roughness transfer in hot rolling depends on oxide scale generated during reheating. When \\ reheating time is increased, the average thickness of oxide scale increases and a relatively rough surface \\ was obtained after hot rolling. Thick oxide scale of 301 steel shows the high lubricative effect. \\ Keywords \\ hot, during, friction, roughness, surface, study, stainless, 301, rolling, steel \\ Disciplines \\ Engineering | Science and Technology Studies \\ Publication Details \\ Wei, D., Jiang, Z., Huang, J., Zhang, A., Shi, X. \& Jiao, S. (2012). Study on surface roughness and friction \\ during hot rolling of stainless steel 301 . Advanced Materials Research, 500 403-409.

\section{Authors} \\ Dongbin Wei, Zhengyi Jiang, Junxia Huang, Aiwen Zhang, Xu Shi, and Sihai Jiao
}

This journal article is available at Research Online: https://ro.uow.edu.au/eispapers/36 


\title{
Pedestrian Lane Detection for Assistive Navigation of Blind People
}

\author{
Manh Cuong Le, Son Lam Phung and Abdesselam Bouzerdoum \\ School of Electrical, Computer and Telecommunications Engineering \\ University of Wollongong, Australia
}

\begin{abstract}
Navigating safely in outdoor environments is a challenging activity for vision-impaired people. This paper is a step towards developing an assistive navigation system for the blind. We propose a robust method for detecting the pedestrian marked lanes at traffic junctions. The proposed method includes two stages: regions of interest (ROI) extraction and lane marker verification. The ROI extraction is performed by using colour and intensity information. A probabilistic framework employing multiple geometric cues is then used to verify the extracted ROI. The experimental results have shown that the proposed method is robust under challenging illumination conditions and obtains superior performance compared to the existing methods.
\end{abstract}

\section{Introduction}

Visual impairment is a significant health problem. The World Health Organization estimates that globally about 285 million people are visually impaired, and 39 million of them are blind [11]. To support the vision impaired navigating safely in outdoor environments, an assistive system should perform several vital tasks such as finding pedestrian lanes, detecting and recognizing traffic obstacles, and sensing dangerous traffic situations.

This paper addresses the task of finding the pedestrian lanes that are indicated by painted markers. Although lane detection for autonomous vehicles has been investigated for several years [2, 4], little work has been done on pedestrian lane detection for blind people. Furthermore, most techniques for detecting pedestrian lanes are designed to find zebra painted patterns $[8,10,3]$.

There are two major classes of approaches for lane detection: edge-based and intensity/colour-based. Edge-based approach use the features of the edges: orientation and magnitude, and a predefined lane model (e.g. straight lines, B-Splines, parabola, or hyperbola) to identify the lane markers. Examples of this approach include $[8,7,6]$. The edge-based approach is simple to implement and has less computational complexity. However, this approach is sensitive to the background noise. In assistive navigation for blind people, since the images are captured at a close range, the cluttered background may strongly affect selecting proper edges representing the lane markers. Alternatively, intensity $[1,4]$ or colour features $[2,5,9]$ are often employed to segment the lane markers. However, intensity information is not discriminative for scenes where the background objects have the similar intensity levels as the lane markers. In addition, the use of only colour is sensitive to lighting condition variations.

In this paper, we propose a new method to detect pedestrian lanes that are marked by two white stripes; these are common cases at traffic intersections (see Fig. 1). Our method includes two stages: ROI extraction and lane marker verification. We employ colour and intensity information for identifying the potential lane markers, and then verify those candidate regions in a probabilistic framework. Geometric features of lane markers are used for this verification. Most existing work on pedestrian lane detection is evaluated based on visual inspection. We address this gap by creating a large data set of images with the ground-truth. This allows performance of detection algorithms to be evaluated rigorously. Our method is evaluated and compared with existing methods on the newly created dataset. Experimental results have shown the robustness of the proposed method.

The remainder of the paper is organized as follows. The proposed method is described in Section 2. Experiments and comparisons are presented in Section 3. The final conclusion is drawn in Section 4.

\section{Proposed Method}

The proposed method has two stages: ROI extraction and lane maker verification. ROI extraction is performed first to segment the candidate lane markers us- 
ing colour and intensity information. From the segmented regions, lane markers are determined by a probabilistic framework.

\subsection{Regions of Interest Extraction}

Compared to the background regions, painted lane markers and road surfaces are distinctive in colour. Painted lane makers have whitish colours while road surfaces have grayish colours of asphalt concrete. Thus, in our method the first step of ROI extraction is pixelwise colour segmentation. In particular, let $p\left(\mathbf{c} \mid \omega_{1}\right)$ and $p\left(\mathbf{c} \mid \omega_{2}\right)$ denote the class-conditional probability density functions ( $p d f$ ) of a colour c (in $R, G, B$ ) for the two classes: lane maker $\omega_{1}$ and non-lane maker $\omega_{2}$. The $p\left(\mathbf{c} \mid \omega_{1}\right)$ and $p\left(\mathbf{c} \mid \omega_{2}\right)$ can be estimated through training. Applying the Bayesian decision rule, a pixel $\mathbf{x}$ is classified as lane maker pixel if

$$
\frac{p\left(C(\mathbf{x}) \mid \omega_{1}\right)}{p\left(C(\mathbf{x}) \mid \omega_{2}\right)} \geq \tau
$$

where $C(\mathbf{x})$ is the colour at $\mathbf{x}$, and $\tau$ is a threshold determined empirically.

Since colour-based segmentation is sensitive to illumination changes, pixels from the road surface with high illumination can be classified as lane marker pixels (see Fig 1). To overcome this issue, intensity information is used to remove such pixels. Specifically, symmetrical local thresholding proposed in [1] is employed. For each detected pixel $\mathbf{x}=(x, y)$ satisfying (1), an adaptive threshold $\gamma$ is calculated as follows:

$$
\gamma=\max \left(\mu_{-}, \mu_{+}\right)+\Delta,
$$

where $\mu_{-}$and $\mu_{+}$denote the mean intensity values of pixels whose $x$-coordinate is in $[x-R, x]$ and $[x, x+R]$ respectively. In our experiment, $R$ is computed relatively to the image width and set to $1 / 4$ of the image width, and $\Delta$ is the difference in the average pixel intensity between the lane marker and road surface obtained from the training data.

Pixels whose the intensity is lower than $\gamma$ are removed. Connected components formed by the remaining pixels are then extracted. After segmentation, regions with small areas are considered as noise and therefore removed. This step results in a set of candidate lane markers $M$. An example of the ROI extraction is shown in Fig. 1.

\subsection{Lane Maker Verification}

In this section, we present a probabilistic method to identify the lane markers given the results of ROI extraction. Instead of verifying individual candidate lane markers, we verify pairs of lane markers using the geometric relationship between two lane markers. For a pair of potential lane markers $\left(m_{i}, m_{j}\right)$, their geometric relationship includes the angle $\varphi_{i j}$, the distance $d_{i j}$ and the vertical overlap $o_{i j}$ between $m_{i}$ and $m_{j}$ as shown in Fig. 2. These cues are informative and discriminative to find the lane region. In particular, the conditional probability of the lane region $\omega_{1}$ for a pair of lane markers $\left(m_{i}, m_{j}\right)$ is computed as

$$
\begin{aligned}
P\left(\omega_{1} \mid m_{i}, m_{j}\right) & =\frac{p\left(m_{i}, m_{j} \mid \omega_{1}\right) P\left(\omega_{1}\right)}{p\left(m_{i}, m_{j}\right)} \\
& =\frac{p\left(\varphi_{i j}, d_{i j}, o_{i j} \mid \omega_{1}\right) P\left(\omega_{1}\right)}{p\left(m_{i}, m_{j}\right)} .
\end{aligned}
$$

We assume that the parameters $\varphi_{i j}, d_{i j}$ and $o_{i j}$ are statistically independent given $\omega_{1}, p\left(m_{i}, m_{j}\right)$ is uniform and $P\left(\omega_{1}\right)$ is constant. Equation (3) can be writen as

$$
P\left(\omega_{1} \mid m_{i}, m_{j}\right) \propto p\left(\varphi_{i j} \mid \omega_{1}\right) p\left(d_{i j} \mid \omega_{1}\right) p\left(o_{i j} \mid \omega_{1}\right),
$$

where $p\left(\varphi_{i j} \mid \omega_{1}\right), p\left(d_{i j} \mid \omega_{1}\right)$ and $p\left(o_{i j} \mid \omega_{1}\right)$ represent the likelihood functions of geometric parameters for pairs of lane markers.

The $p\left(\varphi_{i j} \mid \omega_{1}\right)$ can be computed from the $p d f$ of the angle $\varphi_{i j}$ between two markers of the true lanes given in the training dataset, using the histogram approach. The orientation of a marker region is estimated using the least-square method. In our experiment, the angles $\varphi_{i j}$ within the range $\left[0^{0}, 180^{\circ}\right]$ are quantised into 18 bins.

The distance $d_{i j}$ between two centres of lane markers relative to the image width, represents the width of a true lane. In this paper, we model the $p\left(d_{i j} \mid \omega_{1}\right)$ using the normal distribution as

$$
p\left(d_{i j} \mid \omega_{1}\right)=\frac{1}{\sigma_{d} \sqrt{2 \pi}} e^{-\frac{\left(d_{i j}-\mu_{d}\right)^{2}}{2 \sigma_{d}^{2}}},
$$

where $\mu_{d}$ and $\sigma_{d}$ are estimated from the training data.

Each component $m_{i}$ can be further represented by its closest bounding box $b_{i}$ (see Fig. 2). The vertical overlap $o_{i j}$ is defined as

$$
o_{i j}=\frac{\min \left(b_{i}^{2}, b_{j}^{2}\right)-\max \left(b_{i}^{1}, b_{j}^{1}\right)}{\max \left(b_{i}^{2}, b_{j}^{2}\right)-\min \left(b_{i}^{1}, b_{j}^{1}\right)},
$$

where $b^{1}$ and $b^{2}$ are the top and bottom coordinates of a bounding box $b$ respectively (see Fig 2 ). Note that the image coordinate is employed, i.e. $(0,0)$ is referred to the left-top corner of the image. Due to the perspective projection, lane markers of a pedestrian lane have high vertical overlapping (see Fig 2). Thus, we model the $p\left(o_{i j} \mid \omega_{1}\right)$ based on the vertical overlapping of detected 

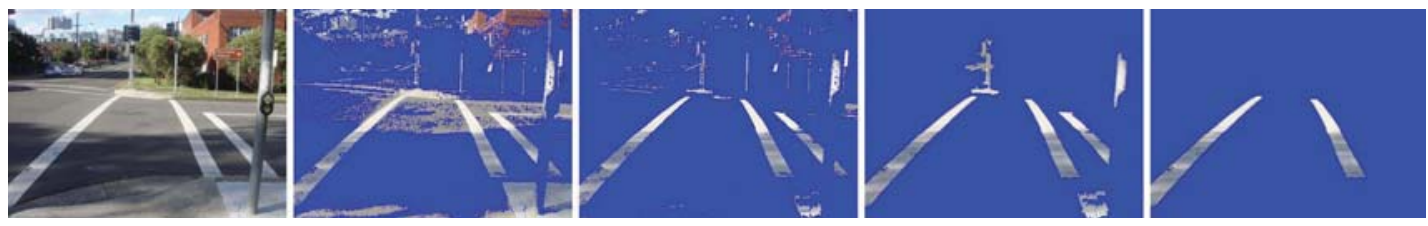

Figure 1. Illustration of the proposed method. From left to right: input image, result of colour-based segmentation, result of local thresholding, results of small components (noise) removal, detected lane markers.

lane markers. In particular, the $p\left(o_{i j} \mid \omega_{1}\right)$ is modeled using the sigmoid function as

$$
p\left(o_{i j} \mid \omega_{1}\right)=\frac{\alpha}{\alpha+e^{-\beta o_{i j}}},
$$

where $\alpha$ and $\beta$ are positive parameters to control the vertical deviation of lane markers. In our experiment, $\alpha$ and $\beta$ are set to 0.2 and 5.0 respectively. Equation (7) indicates that the higher is the overlapping the higher is $p\left(o_{i j} \mid \omega_{1}\right)$.

Finally, the best candidate markers $m_{i}^{*}, m_{j}^{*}$ can be obtained from given a set of candidate lane markers $M$ as

$$
\left(m_{i}^{*}, m_{j}^{*}\right)=\underset{m_{i}, m_{j} \in M}{\arg \max } P\left(\omega_{1} \mid m_{i}, m_{j}\right) .
$$

An result example of lane marker verification is illustrated in Fig. 1.

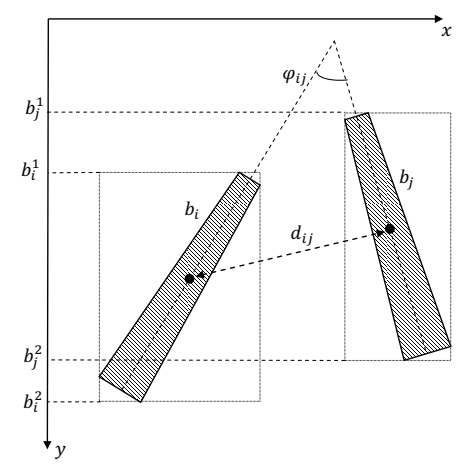

Figure 2. Geometric relationship of two lane markers (shaded areas).

\section{Experimental Results}

\subsection{Image Data and Evaluation Measure}

To evaluate pedestrian lane detection algorithms, we acquired a large dataset of images, taken at traffic cross- ings with different backgrounds, times of day, and weather conditions. We created the ground-truth by manually annotating the image coordinates of pedestrian lane markers. Each lane marker was represented by a polygon. Lane regions then can be obtained from annotated markers. Overall, 1000 images were collected. We used 600 images for training and the remaining images for testing.

Given a ground-truth lane region $\mathbf{R}_{g}$ and a detected lane region $\mathbf{R}_{d}$, the matching score between these two regions is defined as

$$
s\left(\mathbf{R}_{g}, \mathbf{R}_{d}\right)=\frac{\left|\mathbf{R}_{g} \cap \mathbf{R}_{d}\right|}{\left|\mathbf{R}_{g} \cup \mathbf{R}_{d}\right|},
$$

where $\cap$ and $\cup$ denote the intersection and union of two regions and $|\mathbf{R}|$ is the area of region $\mathbf{R}$.

A detected lane region $\mathbf{R}_{d}$ is considered as correct if there exists a ground-truth lane region $\mathbf{R}_{g}$ that satisfies $s\left(\mathbf{R}_{g}, \mathbf{R}_{d}\right) \geq \theta$, where $\theta$ is an evaluation threshold which is set of 0.5 in our experiment.

Two evaluation measures are computed:

- Recall is the percentage of the ground-truth lane regions that are detected correctly by machine.

- Precision is the ratio between the number of the lane regions detected correctly and the total number of machine-generated lane regions.

\subsection{Results}

We implemented the proposed method using Matlab and evaluated it on the newly created dataset. The average processing time for an image of $300 \times 400$ pixels is about $0.83 \mathrm{~s}$, but it could be further optimized. Sample visual results of pedestrian lane detection are presented in Fig. 3. By combining colour and local intensity information, the proposed method detects correctly pedestrian marked lanes in different illumination and weather conditions (sunny, cloudy, strong shadows, times of day). 


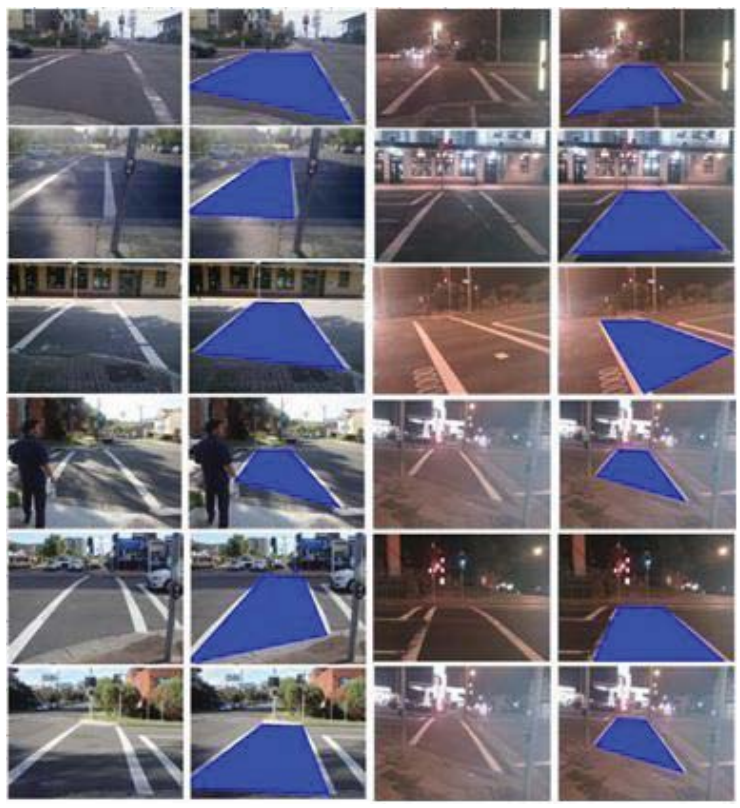

Figure 3. Visual results of lane detection. Columns 1 and 3: input images. Columns 2 and 4: detected pedestrian lane regions.

For comparison purposes, we implemented the Hough transform-based method (HT), which is commonly used in lane detection, e.g. [7]. Another method used for the comparison is the work of Lee and Cho [5] using colour information to extract lane marker pixels and edge orientation information to estimate lane boundaries. The detection performance of these methods are summarised in Table 1.

Table 1. Comparison of algorithms for pedestrian lane detection.

\begin{tabular}{|l|c|c|c|}
\hline Methods & HT & Lee and Cho & Our method \\
\hline Recall (\%) & 72.9 & 75.3 & 91.6 \\
\hline Precision (\%) & 74.3 & 76.5 & 93.5 \\
\hline
\end{tabular}

The proposed method detects correctly $91.6 \%$ of the ground-truth lane regions, whereas the Hough transform method and the algorithm of Lee and Cho can detect only $72.9 \%$ and $75.3 \%$, respectively. Furthermore, our method archives the precision of $93.5 \%$. In comparison, the Hough transform and Lee and Cho methods can obtain the precision of only $74.3 \%$ and $76.5 \%$, respectively.

\section{Conclusion}

This paper proposed a method for robust detection of pedestrian marked lanes at traffic crossings. The proposed method employed colour and intensity information in extracting the candidate markers and verified the extracted markers in a probabilistic framework. Multiple geometric cues were used for the verification. The proposed method was evaluated and compared with existing approaches. Experimental results have shown the potential of the method in challenging environmental conditions.

\section{References}

[1] P. Charbonnier, F. Diebolt, Y. Guillard, and F. Peyret. Road markings recognition using image processing. In IEEE Conf. on Intelligent Transportation System, pages 912-917, 1997.

[2] H. Cheng, B. Jeng, P. Tseng, and K. C. Fan. Lane detection with moving vehicles in the traffic scenes. IEEE Trans. on Intelligent Transportation Systems, 7(4):571582, 2006.

[3] V. Ivanchenko, J. Coughlan, and S. Huiying. Detecting and locating crosswalks using a camera phone. In IEEE Conf. on Computer Vision and Pattern Recognition, pages 1-8, 2008.

[4] Z. W. Kim. Robust lane detection and tracking in challenging scenarios. IEEE Trans. on Intelligent Transportation Systems, 9(1):16-26, 2008.

[5] J. Lee and J. Cho. Effective lane detection and tracking method using statistical modeling of color and lane edge-orientation. In International Conference on Computer Sciences and Convergence Information Technology, pages 1586-1591, 2009.

[6] Q. Li, N. Zheng, and H. Cheng. Springrobot: a prototype autonomous vehicle and its algorithms for lane detection. IEEE Trans. on Intelligent Transportation Systems, 5(4):300-308, 2004.

[7] D. Schreiber, B. Alefs, and M. Clabian. Single camera lane detection and tracking. In IEEE Conf. on Intelligent Transportation Systems, pages 302-307, 2005.

[8] S. Se. Zebra-crossing detection for the partially sighted. In IEEE Conf. on Computer Vision and Pattern Recognition, pages 211-217, 2000.

[9] T. Y. Sun, S. Tsai, and V. Chan. Hsi color model based lane-marking detection. In IEEE Conf. on Intelligent Transportation Systems, pages 1168-1172, 2006.

[10] M. S. Uddin and T. Shioyama. Bipolarity and projective invariant-based zebra-crossing detection for the visually impaired. In IEEE Conf. on Computer Vision and Pattern Recognition, pages 22-30, 2005.

[11] WHO. Visual impairment and blindness. Technical Report Fact Sheet No. 282, 2011. 